This item was submitted to Loughborough's Research Repository by the author.

Items in Figshare are protected by copyright, with all rights reserved, unless otherwise indicated.

\title{
Forward masking of amplitude modulation across ears and its tuning in the modulation domain
}

\section{PLEASE CITE THE PUBLISHED VERSION}

https://doi.org/10.1121/10.0003598

\section{PUBLISHER}

Acoustical Society of America (ASA)

VERSION

AM (Accepted Manuscript)

\section{PUBLISHER STATEMENT}

Copyright (2021) Acoustical Society of America. This article may be downloaded for personal use only. Any other use requires prior permission of the author and the Acoustical Society of America. The following article appeared in The Journal of the Acoustical Society of America, 149 (3), pp.1764-1771 (2021); doi: 10.1121/10.0003598 and may be found at https://asa.scitation.org/doi/10.1121/10.0003598.

\section{LICENCE}

\section{All Rights Reserved}

\section{REPOSITORY RECORD}

Fullgrabe, Christian, Aleksander Sęk, and Brian CJ Moore. 2021. "Forward Masking of Amplitude Modulation Across Ears and Its Tuning in the Modulation Domain". Loughborough University. https://hdl.handle.net/2134/14233955.v1. 
1 Forward masking of amplitude modulation across ears and its tuning in the modulation

2 domain

3

4 Christian Füllgrabea)

5 School of Sport, Exercise and Health Sciences, Loughborough University,

6 Ashby Road, Loughborough LE11 3TU, United Kingdom

7

$8 \quad$ Aleksander Sęk ${ }^{b}$ ) and Brian C.J. Moore

9 Cambridge Hearing Group, Department of Experimental Psychology, University of

10 Cambridge, Downing Street, Cambridge CB2 3EB, United Kingdom

11

12

13 Submitted to the Journal of the Acoustical Society of America

14

15 Running heading: Contralateral modulation forward masking

16

17

18

${ }^{\text {a) Electronic mail: c.fullgrabe@1boro.ac.uk }}$

19 b) Also at Department of Acoustics, Faculty of Physics, Adam Mickiewicz University, 85

20 Umultowska, 61-614 Poznan, Poland

21 


\section{Abstract}

23 Frequency selectivity in the amplitude modulation (AM) domain has been demonstrated

24 using both simultaneous AM masking and forward AM masking. This has been explained

25 using the concept of a modulation filter bank (MFB). Here we assessed whether the MFB

26 occurs before or after the point of binaural interaction in the auditory pathway by using

27 forward masking in the AM domain in an ipsilateral condition (masker AM and signal AM

28 applied to the left ear with an unmodulated carrier in the right ear) and a contralateral

29 condition (masker AM applied to the right ear and signal AM applied to the left ear). The

30 carrier frequency was $8 \mathrm{kHz}$, the signal $\mathrm{AM}$ frequency, $f_{\mathrm{s}}$, was 40 or $80 \mathrm{~Hz}$, and the masker

31 AM frequency ranged from 0.25 to 4 times $f_{\mathrm{s}}$. Contralateral forward AM masking did occur,

32 but it was smaller than ipsilateral AM masking. Tuning in the AM domain was slightly

33 sharper for ipsilateral than for contralateral masking, perhaps reflecting confusion of the

34 signal and masker AM in the ipsilateral condition when their AM frequencies were the same.

35 The results suggest that there might be an MFB both before and after the point in the auditory

36 pathway where binaural interaction occurs.

38 PACS numbers: 43.66.Mk, 43.66.Dc, 43.66.Ba 
I. INTRODUCTION

It has been proposed that the outputs of the (peripheral) auditory filters are fed to an array of overlapping bandpass filters tuned to different envelope modulation frequencies, based on both physiological data (Schreiner and Langner, 1988) and psychophysical data (Kay, 1982; Martens, 1982; Dau et al., 1997a; 1997b; Ewert and Dau, 2000; Ewert et al., 2002; Verhey et al., 2003). This array of filters is usually called a "modulation filter bank" (MFB), and it is assumed that the MFB mediates at least some aspects of the perception of amplitude modulation (AM). Evidence supporting the existence of an MFB comes from experiments involving the detection of signal AM in the presence of masker AM. These experiments show frequency selectivity in the AM domain (Bacon and Grantham, 1989; Houtgast, 1989; Ewert et al., 2002). Frequency selectivity in the AM domain has also been demonstrated in experiments using AM forward masking, where the signal modulation is presented after the masker modulation (Wojtczak and Viemeister, 2005; Moore et al., 2009; Wojtczak et al., 2011). In addition, listeners have some ability to "hear out" the sinusoidal components of a multi-component modulator, provided that the modulator components are widely spaced in frequency (Sęk and Moore, 2003; 2006). It is not clear whether the MFB is located before or after the level in the auditory system where binaural interaction occurs. The present experiment addressed this issue by assessing whether AM forward masking occurs when the masker AM is presented to one ear and the signal AM is presented to the other ear. This is referred to as contralateral modulation masking. Such masking was found to occur, and its tuning in the modulation domain was quantified and compared with that obtained when the masker and signal AM were presented to the same ear.

Thompson and Dau (2008) suggested two possible ways in which frequency selectivity in the modulation domain could be incorporated into models of binaural processing. In the

63 first way, the MFB is applied to the output of each monaural peripheral auditory filter before the input to the binaural processor. In the second way, the inputs of the peripheral auditory filters are combined in the binaural processor and MFBs are applied both to the output of the 66 binaural processor and (separately) to the output of each monaural peripheral auditory filter 67 (see their figure 9). 

neurons tuned to AM frequency in the inferior colliculus, a nucleus that is located in the auditory pathway after the point where binaural interaction first occurs, which is the superior olive (Palmer, 1995). If the neurons found by Langner and Schreiner form the basis for the MFB, then contralateral modulation masking would be expected to occur. On the other hand, neurons tuned to AM frequency have been also been found in the cochlear nucleus, which is located in the auditory pathway before the point where binaural interaction occurs (Palmer, 1995; Joris et al., 2004; Sayles et al., 2013). If the MFB is located before the point of binaural interaction, such that there is a separate MFB for the left and right ears, then little or no contralateral forward masking would be expected to occur, since the output of the MFB on the side receiving the signal AM would be unaffected by the presence of masker AM in the opposite ear.

Psychophysical evidence for binaural interaction in the processing of AM comes from studies showing that human listeners are sensitive to the interaural phase of the envelope of AM sounds for modulation frequencies up to several hundred Hertz. For example, sounds can be lateralized on the basis of interaural envelope delay, for envelope rates up to at least 400 Hz (Henning, 1974; Neutzel and Hafter, 1981; Bernstein and Trahiotis, 2002). However, this might not depend on processing in an MFB, since there is evidence from modulation forward masking that the center frequencies of the modulation filters extend only up to about 100-120 Hz (Moore et al., 2009).

A second line of evidence for binaural interaction in the processing of AM comes from a study of McFadden and Pasanen (1975). They described an analog of binaural beats (Licklider et al., 1950) in the AM domain. A high-frequency two-tone complex was presented to each ear, each complex producing beats with a rate equal to the frequency difference between the two tones. Subjects were required to distinguish between stimuli for which the beat rate was the same at the two ears (e.g., $50 \mathrm{~Hz})$ and for which it was slightly different at the two ears (e.g., $50 \mathrm{~Hz}$ in one ear and $51 \mathrm{~Hz}$ in the other). When the difference

95 in beat rate was $5 \mathrm{~Hz}$ or less, subjects performed well above chance. Subjects reported hearing a fluctuation at a rate corresponding to the difference in beat rate between the two 
ears: $1 \mathrm{~Hz}$ in the example given above. The effect occurred in the presence of an intense lowpass noise and at low levels, so it cannot be explained by combination tones. Related effects showing binaural interaction in the AM domain have been reported by Grantham (1984), Bernstein and Trahiotis (1996), and Sęk et al. (2010). However, again it is not clear whether these effects depend on the processing of the AM in a MFB.

In the present study we sought to determine whether contralateral forward masking in the AM domain occurs, and, if so, to quantify its tuning in the AM domain and to compare it with that obtained in monaural forward masking (Wojtczak and Viemeister, 2005; Moore et al., 2009).

\section{METHOD}

\section{A. Subjects}

Three subjects (two male, one female) were recruited from the student population of the University of Cambridge, and paid for their services. All had audiometric thresholds $\leq 15 \mathrm{~dB}$ hearing level (HL) for both ears at octave frequencies between 0.25 and $8 \mathrm{kHz}$, and at 3 and 6 kHz. Their ages ranged from 22 to 37 years (mean age $=28$ years; standard deviation, $\mathrm{SD}=8$ years). All subjects provided informed written consent and the study was approved by the Cambridge Research Ethics committee.

\section{B. Stimuli}

Stimuli were generated digitally using a Tucker-Davis-Technologies (TDT, Alachua, FL) system II DD1 digital-to-analog converter with 16-bit resolution and a 50-kHz sampling rate. The stimuli were then attenuated (TDT PA4), passed through a headphone buffer (TDT HB6), and routed to a double-walled sound-attenuating booth, where they were passed through a final manual attenuator (Hatfield 2125, Hatfield, UK). Stimuli were delivered via Sennheiser (Wedemark, Germany) HD580 headphones. A PC controlled the stimulus generation, experimental procedure, and data acquisition.

The stimuli were chosen to be similar to those used by Moore et al. (2009). The carrier was an 8-kHz sinusoid with an overall duration of approximately $650 \mathrm{~ms}$ (the exact duration 
126 depended on the masker AM frequency, as described below), including 20-ms raised-cosine

127 onset and offset ramps. This high carrier frequency was chosen so that any spectral sidebands

128 produced by the AM would not be resolved in the auditory system (Kohlrausch et al., 2000;

129 Moore and Glasberg, 2001). In each trial, two sequential carrier bursts, separated by a 415-ms

130 silent interval, were presented to both ears at a level $60 \mathrm{~dB}$ above the mean absolute threshold

131 for the two ears at $8 \mathrm{kHz}$. One of the bursts contained the signal AM and the other did not.

132 Figure 1 shows the envelopes of the stimuli for the "signal" interval. In one condition (Fig.

133 1a), the "absolute threshold" for detecting the signal AM was measured. In this context,

134 "absolute threshold" refers to the threshold for detecting the signal AM in the absence of

135 masker AM. In one randomly chosen interval, both ears received an unmodulated carrier. In

136 the other interval, the right ear received an unmodulated carrier and the left ear received a

137 carrier with a 50-ms-long signal portion whose amplitude was sinusoidally modulated. The

138 signal AM was introduced at least $540 \mathrm{~ms}$ after the onset of the carrier, and always started

139 and ended at positive-going zero-crossings. The carrier was unmodulated and at full

140 amplitude for $40 \mathrm{~ms}$ after the end of the signal modulation and then the unmouldated carrier

141 was ramped off over $20 \mathrm{~ms}$. The depth of the signal modulation, $m$, was varied adaptively to

142 determine the threshold, as described in section II.C. 
(a) No AM masker
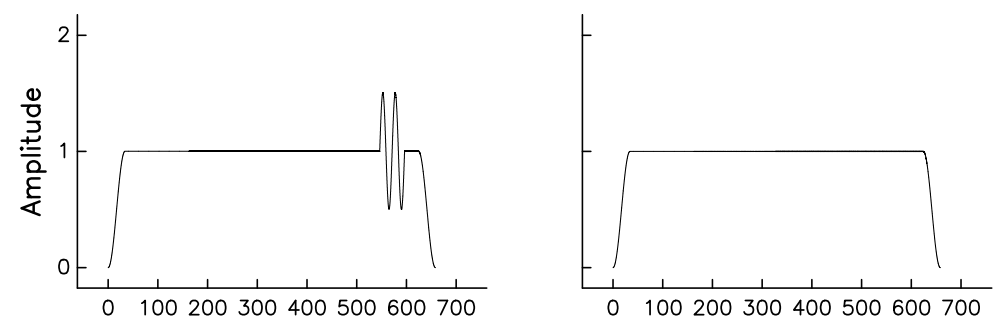

(b) Ipsilateral AM masker

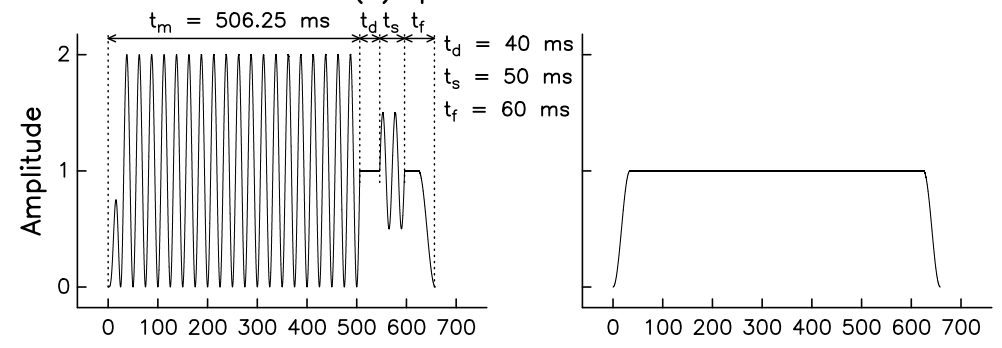

(c) Contralateral AM masker
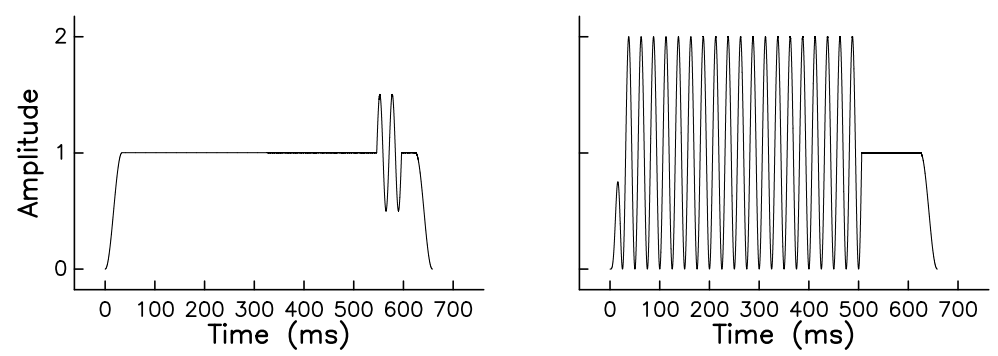

145 FIG. 1. Example of envelopes of $8-\mathrm{kHz}$ carriers delivered to the left and the right ears for a

146 signal interval. The 50-ms signal AM (duration $\mathrm{t}_{\mathrm{s}}$ ) was applied to the carrier presented to the

147 left ear. In one condition, only the signal AM was present (panel a). In the other conditions,

148 the signal AM was preceded by masker AM (duration $\mathrm{t}_{\mathrm{m}}$, AM depth $m=1$ ), applied either to

149 the same carrier as the signal AM (panel b; ipsilateral AM masker) or to the carrier presented

150 to the opposite (i.e., right) ear (panel c; contralateral AM masker). The delay between the end

151 of the masker AM and the start of the signal AM (duration $t_{d}$ ) was 40 ms. The AM depth of

152 the signal was adaptively varied (here shown at $m=0.5$ ).

153 
modulation frequency, $f_{\mathrm{m}}$, was applied to the carrier in one ear in both intervals; this ear was the left for ipsilateral AM masking and the right for contralateral AM masking. No masker AM was applied to the other ear. The masker AM was applied as soon as the carrier was turned on, with a starting phase of $270^{\circ}$, i.e., at its most negative value. The masker AM ended at a positive-going zero crossing ( $0^{\circ}$ phase $)$. The duration of the masker AM was chosen to be close to $500 \mathrm{~ms}$ (see $\mathrm{t}_{\mathrm{m}}$ in Fig. 1b). To determine its exact duration, the masker AM period in ms, $P_{\mathrm{m}}$, was divided into 500, and the result rounded to the nearest integer, $N$. The duration of the masker AM was then computed as:

$$
\left(N \times P_{\mathrm{m}}\right)+0.25 P_{\mathrm{m}}
$$

The end of the masker AM and the start of the signal AM were always separated by $40 \mathrm{~ms}$ (see $t_{d}$ in Fig. 1b). The depth of the masker AM was $m=1$ (Fig. 1b, c).

Signal modulation frequencies, $f_{\mathrm{s}}$, of 40 and $80 \mathrm{~Hz}$ were used, resulting in two and four cycles, respectively, of signal AM. For $f_{\mathrm{s}}=40 \mathrm{~Hz}$, the aprroximately half-octave spaced masker AM frequencies, $f_{\mathrm{m}}$, were $10,14,20,28,40,57,80,113$, and $160 \mathrm{~Hz}$; for $f_{\mathrm{s}}=80 \mathrm{~Hz}$, the values of $f_{\mathrm{m}}$ were $20,28,40,57,80,113,160,226$, and $320 \mathrm{~Hz}$.

\section{Procedure}

First, absolute thresholds for detecting the unmodulated carrier were assessed separately for each ear, using a two-interval, two-alternative forced-choice procedure. In each trial, two intervals, marked by lights on a response box, were presented successively with an inter-stimulus interval of $500 \mathrm{~ms}$. One of the intervals selected at random, contained a $8-\mathrm{kHz}$ pure tone with a duration of $650 \mathrm{~ms}$, including 20-ms raised-cosine rise decay ramps; the other interval was silent. The task was to indicate via a button press which interval contained the tone. Visual feedback as to the correct interval was provided after response entry, and the next trial was initiated automatically after $500 \mathrm{~ms}$. The starting level of the tone was set to be well above the audiometric threshold. A three-down, one-up rule was used to adaptively vary the level to estimate the value necessary for $79.4 \%$-correct detection (Levitt, 1971). The step size started at $4 \mathrm{~dB}$, and was reduced to $2 \mathrm{~dB}$ after two reversals and to $1 \mathrm{~dB}$ after two more reversals. After 12 reversals, the run was terminated and the mean value of the signal level at 
184 last eight reversal points was taken as the threshold estimate. For each ear, three threshold

185 estimates were obtained and their arithmetic mean was taken as the final estimate of absolute

186 threshold. For the main experiment, the carrier level was set to be $60 \mathrm{~dB}$ higher than the mean

187 absolute threshold across the two ears.

188 Then, AM detection thresholds were measured in both the absence and presence of

189 masker AM. The same adaptive procedure as described above was used to estimate the signal

190 AM depth (expressed as $20 \log _{10} m$ ) required to achieve $79.4 \%$ correct. The starting AM depth

191 was set at least $8 \mathrm{~dB}$ above the threshold obtained during practice, or, if that yielded an AM

192 depth greater than $0 \mathrm{~dB}$ (i.e., an over-modulated stimulus), it was set to $0 \mathrm{~dB}$. If the adaptive

193 procedure called for a modulation depth greater than $0 \mathrm{~dB}$, it was limited to $0 \mathrm{~dB}$. If this

194 occurred more than three times after the fourth reversal, the run was aborted and no estimate

195 was computed. This only happened once for one subject. Also, when the SD of the AM

196 depths at the last eight turn points was $\geq 3 \mathrm{~dB}$, the estimate for that run was discarded, and

197 the run was repeated. For each condition, at least four valid threshold estimates were obtained

198 for each subject. When the SD of the threshold estimates across runs was $\geq 3 \mathrm{~dB}$, an

199 additional estimate was obtained; this was repeated, if necessary, until up to six estimates

200 were measured. Threshold estimates more than $5 \mathrm{~dB}$ away from the final mean threshold

201 were regarded as outliers and discarded (1\% of all runs). The final estimate was taken as the

202 average across all valid runs.

203 The ipsilateral and contralateral masker conditions were assessed in separate sessions.

204 Subjects S1 and S3 first completed the contralateral, and then the ipsilateral conditions,

205 whereas S2 did the reverse. Unmasked AM detection thresholds for one signal AM frequency

206 were measured at the beginning of each session, before measuring forward masking

207 thresholds using all masker AM frequencies, in random order. Only one signal AM frequency

208 was used in each session, and usually each masker AM frequency was used twice in each

209 session. At least four sessions were used for each subject.

210 All subjects were highly trained, with over $30 \mathrm{~h}$ of experience with AM forward

211 masking, due to their participation in a previous study using similar stimulus parameters

212 (Moore et al., 2009). However, since only monaural stimuli were used in that study, all 
213 listeners received a further 3-4 h of practice using various signal-masker AM combinations.

\section{III. RESULTS}

216 The results are shown in Fig. 2. Individual and mean AM depths at threshold are plotted

217 in terms of $20 \log _{10} m$ (left ordinate) and $m$ expressed as a percentage (right ordinate). Signal

218 AM detection thresholds for $40-\mathrm{Hz}$ and $80-\mathrm{Hz}$ AM in the absence of masker AM are shown

219 by the dashed and continuous horizontal lines, respectively. The average AM threshold was

$220-21 \mathrm{~dB}(9 \%)$ for the $40-\mathrm{Hz}$ signal and $-23 \mathrm{~dB}(7 \%)$ for the $80-\mathrm{Hz}$ signal. These thresholds are

221 about $10 \mathrm{~dB}$ higher (i.e, worse) than AM thresholds reported in the literature for high-

222 frequency pure-tone carriers (Kohlrausch et al., 2000; Moore and Glasberg, 2001; Füllgrabe

223 and Lorenzi, 2003). This discrepancy and the fact that thresholds in the present study were 2

$224 \mathrm{~dB}$ higher for the $40-\mathrm{Hz}$ than for the $80-\mathrm{Hz}$ AM can probably be explained mainly by the

225 small numbers of AM cycles in the signal portion of the stimulus, which was 2 for the $40-\mathrm{Hz}$

226 AM and 4 for the 80-Hz AM (Sheft and Yost, 1990; Lee and Bacon, 1997). An additional

227 factor is that the signal AM was applied to the carrier in one ear only; the carrier in the other

228 ear was unmodulated. In most previous studies, the signals were diotic, with the signal AM

229 presented to both ears (Kohlrausch et al., 2000). 


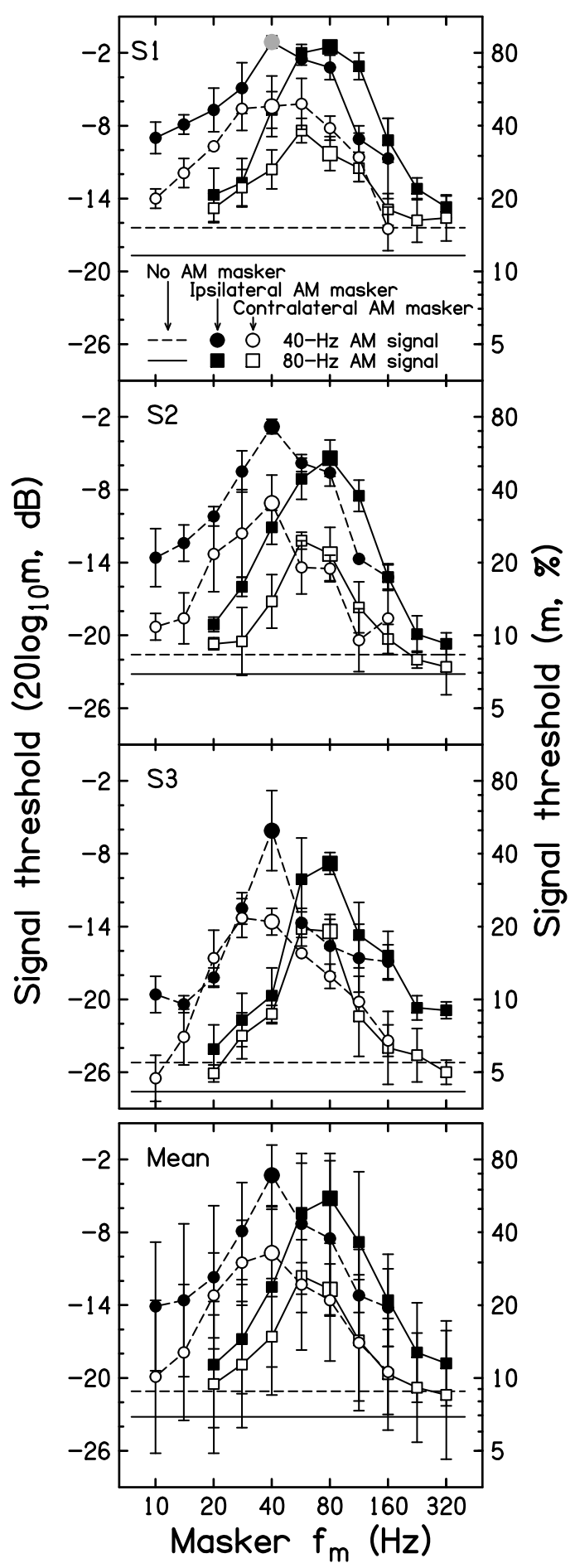

232 FIG. 2. Individual and mean results. The signal AM depth at threshold [expressed as

$23320 \log _{10} m$ (left ordinate) and percent (right ordinate)] for 40-Hz (circles) and $80-\mathrm{Hz}$ (squares)

234 signal $\mathrm{AM}$ is plotted as a function of the AM frequency $\left(f_{\mathrm{m}}\right)$ of the $\mathrm{AM}$ masker. Results for

235 the ipsilateral and contralateral conditions are shown by filled and open symbols, 
236

237

238

239

240

241

242

243

244

245

246

247

248

249

250

251

252

253

254

255

256

257

258

259

260

261

262

263

264

respectively. Larger symbols denote conditions in which the masker and signal had the same AM frequency. A condition in which one threshold run was aborted due to the overmodulation rule is flagged by the light grey symbol; the plotted data point represents the average of completed runs. Error bars in the top three panels indicate \pm 1 SD across repeated measures. Error bars in the bottom panel indicate \pm 1 SD across subjects. Signal detection thresholds without the forward AM masker are indicated by broken (40-Hz signal) and continuous (80-Hz signal) horizontal lines.

In Fig. 2, the different symbols show forward-masked AM detection thresholds as a function of $f_{\mathrm{m}}$ : circles denote thresholds for the 40-Hz signal, while squares denote those for the $80-\mathrm{Hz}$ signal. Filled symbols indicate the ipsilateral condition, with the masker AM applied to the same carrier as the signal modulation. As found previously for similar stimuli, AM forward masking occurred and it showed tuning in the AM frequency domain, with the highest thresholds occurring for masker AM frequencies close to the signal AM frequency (Wojtczak and Viemeister, 2005; Moore et al., 2009). Open symbols indicate the contralateral condition, with the masker AM applied to the carrier in the opposite ear to that with the signal AM. It is obvious that contralateral AM forward masking did occur, although it was somewhat smaller for the contralateral than for the ipsilateral condition. Averaged across all masker AM frequencies for both signal AM frequencies, the amount of AM masking (masked AM threshold minus absolute AM threshold) was $10.3 \mathrm{~dB}$ for the ipsilateral condition and $5.8 \mathrm{~dB}$ for the contralateral condition.

To assess the effect of ear of masker presentation (ipsilateral versus contralateral) on AM thresholds and to assess whether frequency selectivity in the AM domain was affected by the signal frequency and by the ear of masker presentation, an analysis of variance (ANOVA) was conducted on the masked AM thresholds with factors ear of presentation, signal AM frequency, and masker AM frequency. For this analysis, the masker AM frequency was divided by the signal AM frequency, i.e., it was expressed relative to the signal AM frequency. The masked AM thresholds sometimes approached the absolute AM detection thresholds for the two lowest and two highest relative masker frequencies. For those cases, 
the AM thresholds were probably limited by the approach to the absolute AM detection threshold rather than by the tuning of the MFB. Since our primary interest was to assess whether frequency selectivity in the AM domain was affected by the ear of masker presentation, the results for these relative masker frequencies were excluded from the analysis. When the condition of sphericity was not met, the Huynh-Feldt correction was applied to the degrees of freedom, but the uncorrected degrees of freedom are reported.

There were, as expected, significant effects of ear of presentation $[F(1,2)=42.7, p=$ $0.023]$ and of relative masker frequency $[F(4,8)=17.7, p=0.012]$. There was a significant effect of signal AM frequency $[F(1,2)=49.3, p=0.012]$, mean masked thresholds being higher for the 40-Hz than for the 80-Hz signal AM frequency. However, the absolute thresholds for AM detection were also higher for $40-\mathrm{Hz}$ than for the $80-\mathrm{Hz}$ signal AM frequency. The mean amount of forward masking (masked threshold minus absolute threshold) was similar for the two AM signal frequencies. The interaction of signal AM frequency and relative masker frequency was significant $[F(4,8)=8.57, p=0.005]$, meaning that the pattern of tuning in the AM domain was different for the two AM signal frequencies. The interaction of ear of presentation and relative masker frequency was significant $[F(4,8)$ $=8.57, p=0.005]$, meaning that the pattern of tuning in the AM domain was different for ipsilateral and contralateral masker presentation. No other interactions were significant.

To quantify the sharpness of the masking patterns, the data were fitted with secondorder Butterworth bandpass filters (Ewert and Dau, 2000), as was done by Wojtczak and Viemeister (2005) and Moore et al. (2009). Such filters are symmetrical on a logarithmic frequency scale, consistent with the shapes of the masking patterns in Fig. 2. The center frequency of each fitted filter was constrained to be equal to the signal frequency. As for the ANOVA described earlier, the data for the two lowest and two highest relative masker frequencies were excluded from the analysis. Table 1 shows the $Q$ values of the fitted filters (center frequency divided by bandwidth at the 3-dB down point) for the individual and mean data. It can be seen that the $Q$ values tend to be smaller, i.e. the filters are less sharp, for contralateral than for ipsilateral presentation. However, an ANOVA with factors signal AM frequency and condition (ipsilateral or contralateral) showed no significant effect of AM 
294 frequency $[F(1,2)=12.5, p=0.071]$ or of condition $[F(1,2)=2.36, p=0.265]$. The

295 interaction was also not significant: $F(1,2)=1.14, p=0.40$.

296 The $Q$ values are similar to but slightly higher than those reported by Wojtczak and

297 Viemeister (2005) for 20, 40, and 80-Hz signal AM and are similar to those reported by

298 Moore et al. (2009) for 80-Hz signal AM.

299

\begin{tabular}{ccccc}
\hline \hline & \multicolumn{2}{c}{ Signal AM frequency $=40 \mathrm{~Hz}$} & \multicolumn{2}{c}{ Signal AM frequency $=80 \mathrm{~Hz}$} \\
\hline Subject & Ipsilateral & Contralateral & Ipsilateral & Contralateral \\
\hline S1 & $0.87(1.5)$ & $0.67(0.7)$ & $1.1(1.1)$ & $0.96(1.7)$ \\
S2 & $1.2(1.4)$ & $1.1(1.6)$ & $1.4(1.4)$ & $1.3(2.0)$ \\
S3 & $2.4(1.2)$ & $0.83(1.0)$ & $1.8(1.8)$ & $1.6(2.4)$ \\
Mean & $1.5(1.3)$ & $0.80(0.7)$ & $1.4(1.0)$ & $1.25(2.0)$ \\
\hline \hline
\end{tabular}

300

301 Table I. $Q$ values of the second-order Butterworth filters fitted to the individual and mean data presented in Fig. 2 for each condition (ipsi- or contralateral) and signal AM frequency (40 or $80 \mathrm{~Hz}$ ). Numbers in parentheses show the root-mean-square error (in dB) of the fits.

\section{DISCUSSION}

Clear forward masking in the AM domain was found when the signal and masker AM were applied to opposite ears. This finding differs from that of Gutschalk et al. (2008), who investigated the effect of adaption to a 60-s long pulse train with an average frequency of 100 $\mathrm{Hz}$ on the subsequent detection of AM. They found that thresholds for detecting sinusoidal AM applied to a noise carrier and presented after the adaptor, were elevated for AM

311 frequencies from 100 to $500 \mathrm{~Hz}$ when the signal AM was presented to the same ear as the 312 adaptor, but not when the signal AM was presented to the opposite ear. It is not clear why 313 AM forward masking using relatively brief masker AM (about $500 \mathrm{~ms}$ in the present study) 314 occurs across ears, but longer-term adaptation to AM does not lead to an effect across ears. It 315 is possible that the lack of contralateral transfer found by Gutschalk et al. (2008) reflects the 
high AM frequency used by them, but we found contralateral AM forward masking for an 80-

$317 \mathrm{~Hz}$ AM masker, and this is only a little lower than the $100-\mathrm{Hz}$ frequency used by Gutschalk et

318 al. (2008). It is noteworthy that adaptation to a frequency-modulated sinusoidal carrier does

319 affect the threshold for detecting frequency modulation applied to a carrier in the opposite ear

320 (Kay and Mathews, 1972).

321 The fact that forward masking in the AM domain was found when the signal and masker AM were applied to opposite ears suggests that part of the forward masking effect occurs higher in the auditory pathway than the earliest point of binaural interaction, which is the superior olive (Palmer, 1995). Given that the contralateral masking showed tuning in the AM domain, and hence presumably reflects the operation of the MFB, the results suggest that the MFB resides at least partly after the point in the auditory pathway where binaural interaction occurs. However, the masked thresholds were lower for contralateral than for ipsilateral forward masking. This could mean that there is more than one MFB, with one MFB occurring after the point of binaural interaction and with independent MFBs for the left and right ears, as for the second of the two models described by Thompson and Dau (2008). If so, this would mean that subjects could not attend exclusively to the outputs of the monaural MFBs, even if this would be the best strategy. Alternatively, the MFB might occur entirely at a higher level than the superior olive, but it may receive inputs from both monaural pathways and binaural pathways.

A relatively central site for the MFB is consistent with the results of Malone et al. (2015). They measured neural responses to AM tones in the auditory cortex of awake squirrel monkeys. They found that presentation of "masker" AM of a given frequency $(4,10,32$, or $96 \mathrm{~Hz}$ ) produced suppression of the firing rate to "signal" AM presented subsequently. The effect showed broad tuning in the AM domain. The masker AM did not affect neural synchrony to the signal AM. Malone et al. (2015) concluded that "modulation-frequencyspecific rate suppression is broadly consistent with the operation of a modulation filterbank" and that "modulation masking may be instantiated in the forebrain" (page 5913). 
case that AM forward masking is governed primarily by the perceptual similarity of the masker AM and signal AM, as appears to be the case for modulation detection interference

347 (MDI) (Yost and Sheft, 1989; Yost et al., 1989; Moore and Shailer, 1992). MDI has also been observed when the masker and signal modulation are presented non-simultaneously (Oxenham and Dau, 2001; Gockel et al., 2002). It has been argued that MDI primarily reflects a form of informational masking (Sheft and Yost, 2007). This may also be the case for AM forward masking. It may be that informational masking is stronger when the masker AM and signal AM are presented to the same ear than when they are presented to opposite ears.

The significant interaction of $f_{\mathrm{s}}$ and relative masker frequency and the significant interaction of ear of presentation and relative masker frequency partly reflect the fact that the masking patterns were somewhat more sharply peaked for the $40-\mathrm{Hz}$ than for the $80-\mathrm{Hz}$ signal frequency, especially for the ipsilateral condition. Note that for S1 the ipsilateral threshold for $f_{\mathrm{m}}=f_{\mathrm{s}}=40 \mathrm{~Hz}$ was probably underestimated because one run was aborted owing to the 0-dB limit imposed on the AM depth. The masking pattern for S1 would have been sharper for $f_{\mathrm{s}}=40 \mathrm{~Hz}$ if this underestimation had not occurred. The relatively sharp peak for the ipsilateral condition for $f_{\mathrm{s}}=40 \mathrm{~Hz}$ may partly reflect confusion of the signal and the masker AM when the two had the same frequency and a similar AM depth; the subject may have heard the signal AM but it may have sounded like the masker AM and it may have been difficult for the subject to decide whether the masker AM had ended and the signal AM had started or whether the masker AM had continued. This is analogous to the confusion effects that have been observed for forward masking in the audio-frequency domain (Terry and Moore, 1977; Moore and Glasberg, 1983; Moore and Glasberg, 1985; Neff, 1986). Wojtczak and Viemeister (2005) assessed the possible existence of confusion in ipsilateral AM forward masking for two subjects who could not perform the task when $f_{\mathrm{m}}=f_{\mathrm{s}}$

$370=40 \mathrm{~Hz}$ and when there was no delay between the end of the masker AM and the start of the signal AM. They used a noise carrier, and investigated the effect of providing a cue to

372 indicate when the masker AM ended. One cue, called the tonal cue, was a 4-kHz sinewave 373 that was presented to the same ear as the noise carrier and was gated synchronously with the 
masker AM. The other cue, called the binaural cue, was an independent sample of the noise carrier that was presented to the contralateral ear and which contained masker AM but not signal AM. The tonal cue led to improvements in the signal AM threshold for delays between the signal and masker AM up to $15 \mathrm{~ms}$, consistent with a confusion effect in the absence of a cue. The binaural cue did not lead to improved signal AM thresholds for any delay. The authors suggested that the binaural cue "may have provided distraction, causing a slight increase in forward masking" for short delays. Wojtczak and Viemeister (2005) concluded that confusion effects were probably small, although they also stated that the cues used may not have been effective in reducing confusion. In any case, the magnitude of confusion effects may depend on whether the carrier is a broadband noise, as used by Wojtczak and Viemeister (2005), or a sinusoid, as used in the present study and by Moore et al. (2009). Performance in the AM forward-masking task when the signal and masker AM have the same frequency may depend on the ability to detect the gap in the AM between the masker and the signal. Thresholds for detecting gaps in AM imposed on a high-frequency sinusoidal carrier are roughly proportional to the AM period (Sęk and Moore, 2002). The AM gap threshold would have been higher for the 40-Hz signal AM than for the $80-\mathrm{Hz}$ signal $\mathrm{AM}$, and this could account for the finding that the ipsilateral masking patterns were sharply peaked for the $40-\mathrm{Hz}$ but not for the $80-\mathrm{Hz}$ signal AM. Confusion effects in forward masking are unlikely when the signal AM and masker AM are presented to opposite ears, which can account for why the masking patterns showed slightly less sharp peaks for contralateral than for ipsilateral presentation. The tuning in the AM domain for the contralateral condition may therefore give a more accurate estimate of the selectivity of the MFB than the tuning in the ipsilateral condition.

\section{CONCLUSIONS}

(1) AM forward masking occurred in the modulation domain when the signal AM was applied to one ear and the masker AM was applied to the other ear (contralateral condition). (2) AM masking in the contralateral condition was smaller than found when the signal AM was applied to the same ear as the masker AM (ipsilateral condition), but in both conditions 
403

404

405

406

407

408

409

410

411

412

413

414

415

416

417

418

419

420

421

422

423

424

425

426

427

428

429

430

431

432

433

the AM forward masking was greatest when the masker AM frequency was similar to the signal AM frequency, reflecting tuning in the AM domain.

(3) The results are consistent with the idea that there is a modulation filter bank that occurs higher in the auditory pathway than the point of binaural interaction.

(4) The masking patterns tended to show sharper peaks in the AM frequency domain for the ipsilateral than for the contralateral condition, perhaps reflecting confusion of the signal and masker AM in the ipsilateral condition when the signal and masker had the same AM frequency. However, the estimated Q values of the masking patterns did not differ significantly for the ipsilateral and contralateral conditions. Testing with a greater number of subjects would be desirable to assess whether selectivity in the AM domain differs for the ipsilateral and contralateral conditions.

\section{ACKNOWLEDGMENTS}

This work was supported by an EU Marie Curie Fellowship and a Wolfson College (Cambridge, UK) Junior Research Fellowship (author CF), an MRC grant (author BM), and a grant from Deafness Research UK (authors BM and AS). We thank two reviewers for helpful comments on an earlier version of this paper.

Bacon, S. P., and Grantham, D. W. (1989). "Modulation masking: effects of modulation frequency, depth, and phase," J. Acoust. Soc. Am. 85, 2575-2580.

Bernstein, L. R., and Trahiotis, C. (1996). "Binaural beats at high frequencies: Listeners' use of envelope-based interaural temporal and intensive disparities," J. Acoust. Soc. Am. 99, 1670-1679.

Bernstein, L. R., and Trahiotis, C. (2002). "Enhancing sensitivity to interaural delays at high frequencies by using "transposed stimuli"," J. Acoust. Soc. Am. 112, 1026-1036.

Dau, T., Kollmeier, B., and Kohlrausch, A. (1997a). "Modeling auditory processing of amplitude modulation. I. Detection and masking with narrowband carriers," J. Acoust. Soc. Am. 102, 2892-2905.

Dau, T., Kollmeier, B., and Kohlrausch, A. (1997b). "Modeling auditory processing of amplitude modulation. II. Spectral and temporal integration," J. Acoust. Soc. Am. 102, 2906-2919. 
434

435

436

437

438

439

440

441

442

443

444

445

446

447

448

449

450

451

452

453

454

455

456

457

458

459

460

461

462

463

464

465

466

467

Ewert, S. D., and Dau, T. (2000). "Characterizing frequency selectivity for envelope fluctuations," J. Acoust. Soc. Am. 108, 1181-1196.

Ewert, S. D., Verhey, J. L., and Dau, T. (2002). "Spectro-temporal processing in the envelope-frequency domain," J. Acoust. Soc. Am. 112, 2921-2931.

Füllgrabe, C., and Lorenzi, C. (2003). "The role of envelope beat cues in the detection and discrimination of second-order amplitude modulation," J. Acoust. Soc. Am. 113, 49-52.

Grantham, D. W. (1984). "Discrimination of dynamic interaural intensity differences," J. Acoust. Soc. Am. 76, 71-76.

Gutschalk, A., Micheyl, C., and Oxenham, A. J. (2008). "The pulse-train auditory aftereffect and the perception of rapid amplitude modulations," J. Acoust. Soc. Am. 123, 935-945.

Henning, G. B. (1974). "Detectability of interaural delay in high-frequency complex waveforms," J. Acoust. Soc. Am. 55, 84-90.

Houtgast, T. (1989). "Frequency selectivity in amplitude-modulation detection," J. Acoust. Soc. Am. 85, 1676-1680.

Joris, P. X., Schreiner, C. E., and Rees, A. (2004). "Neural processing of amplitudemodulated sounds," Physiol. Rev. 84, 541-577.

Kay, R. H. (1982). "Hearing of modulation in sounds," Physiol. Rev. 62, 894-975.

Kay, R. H., and Mathews, D. R. (1972). "On the existence in human auditory pathways of channels selectively tuned to the modulation present in frequency-modulated tones," J. Physiol. 225, 657-677.

Kohlrausch, A., Fassel, R., and Dau, T. (2000). "The influence of carrier level and frequency on modulation and beat-detection thresholds for sinusoidal carriers," J. Acoust. Soc. Am. 108, 723-734.

Langner, G., and Schreiner, C. E. (1988). "Periodicity coding in the inferior colliculus of the cat. I. Neuronal mechanisms," J. Neurophysiol. 60, 1799-1822.

Lee, J., and Bacon, S. P. (1997). "Amplitude modulation depth discrimination of a sinusoidal carrier: Effect of stimulus duration," J. Acoust. Soc. Am. 101, 3688-3693.

Levitt, H. (1971). "Transformed up-down methods in psychoacoustics," J. Acoust. Soc. Am. 49, 467-477.

Licklider, J. C., Webster, J. C., and Hedlun, J. M. (1950). "On the frequency limits of binaural beats," J. Acoust. Soc. Am. 22, 468-473.

Malone, B. J., Beitel, R. E., Vollmer, M., Heiser, M. A., and Schreiner, C. E. (2015). "Modulation-frequency-specific adaptation in awake auditory cortex," J. Neurosci. 35, 5904-5916. 
468

469

470

471

472

473

474

475

476

477

478

479

480

481

482

483

484

485

486

487

488

489

490

491

492

493

494

495

496

497

498

499

Martens, J.-P. (1982). "A new theory for multi-tone masking," J. Acoust. Soc. Am. 72, $397-$ 405.

McFadden, D., and Pasanen, E. G. (1975). "Binaural beats at high frequencies," Science 190, 394-396.

Moore, B. C. J., Füllgrabe, C., and Sęk, A. (2009). "Estimation of the center frequency of the highest modulation filter," J. Acoust. Soc. Am. 125, 1075-1081.

Moore, B. C. J., and Glasberg, B. R. (1983). "Growth of forward masking for sinusoidal and noise maskers as a function of signal delay: implications for suppression in noise," J. Acoust. Soc. Am. 73, 1249-1259.

Moore, B. C. J., and Glasberg, B. R. (1985). "The danger of using narrowband noise maskers to measure suppression," J. Acoust. Soc. Am. 77, 2137-2141.

Moore, B. C. J., and Glasberg, B. R. (2001). "Temporal modulation transfer functions obtained using sinusoidal carriers with normally hearing and hearing-impaired listeners," J. Acoust. Soc. Am. 110, 1067-1073.

Neff, D. L. (1986). "Confusion effects with sinusoidal and narrowband-noise forward maskers," J. Acoust. Soc. Am. 79, 1519-1529.

Neutzel, J. M., and Hafter, E. R. (1981). "Lateralization of complex waveforms: Spectral effects," J. Acoust. Soc. Am. 69, 1112-1118.

Palmer, A. R. (1995). "Neural signal processing," in Hearing, edited by B. C. J. Moore (Academic Press, San Diego), pp. 75-121.

Sayles, M., Fullgrabe, C., and Winter, I. M. (2013). "Neurometric amplitude-modulation detection threshold in the guinea-pig ventral cochlear nucleus," J. Physiol. 591, 34013419.

Schreiner, C. E., and Langner, G. (1988). "Periodicity coding in the inferior colliculus of the cat. II. Topographical organization," J. Neurophysiol. 60, 1823-1840.

Sęk, A., Glasberg, B. R., and Moore, B. C. J. (2010). "The origin of binaural interaction in the modulation domain," J. Acoust. Soc. Am. 127, 2451-2460.

Sęk, A., and Moore, B. C. J. (2002). "Mechanisms of modulation gap detection," J. Acoust. Soc. Am. 111, 2783-2792.

Sęk, A., and Moore, B. C. J. (2003). "Testing the concept of a modulation filter bank: The audibility of component modulation and detection of phase change in three-component modulators," J. Acoust. Soc. Am. 113, 2801-2811. 
500 Sęk, A., and Moore, B. C. J. (2006). "Perception of amplitude modulation by hearingimpaired listeners: The audibility of component modulation and detection of phase change in three-component modulators," J. Acoust. Soc. Am. 119, 507-514.

Sheft, S., and Yost, W. A. (1990). "Temporal integration in amplitude modulation detection," J. Acoust. Soc. Am. 88, 796-805.

Terry, M., and Moore, B. C. J. (1977). "'Suppression' effects in forward masking," J. Acoust. Soc. Am. 62, 781-784.

Thompson, E. R., and Dau, T. (2008). "Binaural processing of modulated interaural level differences," J. Acoust. Soc. Am. 123, 1017-1029.

Verhey, J. L., Ewert, S. D., and Dau, T. (2003). "Modulation masking produced by complex tone modulators," J. Acoust. Soc. Am. 114, 2135-2146.

511 Wojtczak, M., Nelson, P. C., Viemeister, N. F., and Carney, L. H. (2011). "Forward masking in the amplitude-modulation domain for tone carriers: psychophysical results and physiological correlates," J. Assoc. Res. Otolaryngol. 12, 361-373.

514 Wojtczak, M., and Viemeister, N. F. (2005). "Forward masking of amplitude modulation: Basic characteristics," J. Acoust. Soc. Am. 118, 3198-3210. 